\title{
TAGUNG
}

\section{Das Europäische Parlament: Funktionswandel und Aufgabenprofile 2014-2019}

\author{
Martina Fürrutter, Regina Tschann und Michael Wolf*
}

Die Analyse der sechsten und der Ausblick auf die nach den Parlamentswahlen beginnende siebte Legislaturperiode 2014-2019 des Europäischen Parlaments waren Anlass für die Fachtagung, in deren Mittelpunkt die Diskussion von Untersuchungen und Trends zum Funktionswandel des Parlaments, seiner Fraktionen, Ausschüsse und der Europarteien sowie hieraus abgeleiteten Aufgabenprofilen in der kommenden Legislaturperiode standen.

Nicht nur als Kontrollinstanz, sondern auch als System- und Politikgestalter konnte das Europäische Parlament seine Kompetenzen, seit seiner Schaffung 1952 als „Parlamentarische Versammlung“, deutlich ausbauen. Seit den ersten Direktwahlen 1979 ist es das einzige unmittelbar gewählte und damit demokratisch legitimierte Organ der Europäischen Union. Im Verlauf des europäischen Einigungs- und Vertragsbildungsprozesses wurde das Parlament immer mehr zum Motor der Unionsentwicklung. Die Diskussion seiner Politikgestaltungs-, Wahl-, Kontroll-, Kommunikationsund Interaktionsfunktion zeigt, dass das Parlament spätestens seit dem Vertrag von Lissabon ein mit dem Ministerrat auf Augenhöhe agierender Akteur ist. Die Wesensmerkmale des Parlaments sind vielfältig: Zu nennen sind seine als Agendasetzer und Mitgesetzgeber gesteigerte Politikgestaltungskompetenz, die Aufwertung der Parlamentswahlen als indirekte Form der Wahl des Kommissionspräsiden-

\section{Das Europäische Parlament - Funktionswandel und Aufgabenprofile 2014-19}

\section{4.-16. Februar 2014, Tutzing}

Gemeinsame Tagung des Arbeitskreises Europäische Integration e.V., der Akademie für Politische Bildung Tutzing und der Universität Innsbruck.

Mit freundlicher Unterstützung der Europäischen Kommission.

\section{Begrüßung und Eröffnung der Tagung}

Dr. Andreas KALINA, Akademie für Politische Bildung Tutzing

Dr. Anja OPITZ, Akademie für Politische Bildung Tutzing

Prof. Dr. Andreas MAURER, Institut für Politikwissenschaft, Universität Innsbruck

Dr. Dietmar NICKEL, Generaldirektor EuropaKolleg Hamburg

Bilanz und Herausforderungen in zentralen Funktionsbereichen

Bilanz des europäischen Parlamentarismus

Dr. Ingo FRIEDRICH, Vizepräsident des Europäischen Parlaments a.D.

Politikgestaltungsfunktionen des Europäischen Parlaments

Prof. Dr. Andreas MAURER, Institut für Politikwissenschaft, Universität Innsbruck

* Mag. Martina Fürrutter, wissenschaftliche Mitarbeiterin am Institut für Politikwissenschaft der Leopold-Franzens-Universität Innsbruck und des Innsbruck Center for European Research (ICER).

Regina Tschann, studentische Mitarbeiterin am Institut für Politikwissenschaft der Leopold-Franzens-Universität Innsbruck und des Innsbruck Center for European Research (ICER).

Michael Wolf, studentischer Mitarbeiter am Institut für Politikwissenschaft der Leopold-Franzens-Universität Innsbruck und des Innsbruck Center for European Research (ICER). 
ten, seine Kontrollfunktionen gegenüber der Europäischen Kommission und dem Europäischen Auswärtigen Dienst, seine Mitwirkungsbefugnisse im Unionshaushalt, das Bemühen um eine verbesserte EU-weite Kommunikation und die verstärkte interparlamentarische Zusammenarbeit im Hinblick auf die Kontrolle des Ratssystems. Seit seiner Schaffung durchlebte das Parlament einen signifikanten, von Höhen und Tiefen gekennzeichneten Entwicklungsprozess. Die Krise der Europäischen Wirtschafts- und Währungsunion, die Debatten zum Transatlantischen Handels- und Investitionsabkommen oder die Auseinandersetzungen über Sicherheitsfragen in der Netzpolitik zeigen, dass diese Entwicklung noch lange nicht an ihr Ende gekommen ist, sondern dass auch in Zukunft viele Herausforderungen auf das Europäische Parlament und die Union im Allgemeinen warten.

\section{Politikgestaltung im Mehrebenensystem}

Nach einer kurzen Einführung, in der die Tagungskonzeption anhand des Funktionsmodells des Europäischen Parlaments im Kontext des Mehrebenenparlamentarismus skizziert wurde, war das erste Panel den Funktionsbereichen des Europäischen Parlaments gewidmet. In seiner Politikgestaltungsfunktion bewegt sich das Europäische Parlament in einem Spannungsfeld zwischen Mitentscheidung, Mitgestaltung und Mitverantwortung. In Folge der konstitutionellen Entwicklung der Europäischen Union ist so nicht nur eine steigende, an vertraglich festgelegten Verfahrensarten gemessene Beteiligung des Europäischen Parlaments im Gesetzgebungsverfahren zu beobachten, sondern gleichzeitig auch eine auf hohem Niveau bleibende Nichtbeteiligung an wichtigen Politiken, zum Beispiel in den Bereichen Währung, Sicherheit und Sozialpolitik. Die simultane Ausweitung derjenigen Politikbereiche, in denen das Parlament im Mitentscheidungsverfahren als Mitgesetzgeber agiert und jener intergouvernementalen Politikfelder, in denen es nicht beteiligt ist, resultieren so in zwei gegenläufigen Tendenzen:
Das Europäische Parlament in der Systemgestaltung der $E U$

Dr. Martin GROSSE HÜTTMANN, Institut für Politikwissenschaft, Eberhard-Karls-Universität Tübingen

Wahl- und Kreationsfunktionen des Europäischen Parlaments

Dr. Dietmar NICKEL, Generaldirektor a.D. des Europäischen Parlaments, Europa-Kolleg Hamburg

Profilbildung im Europäischen Parlament

Langfristige Trends der Wahlen zum Europäischen Parlament

Prof. Dr. Oskar NIEDERMAYER, Otto-Suhr-Institut für Politikwissenschaft, Freie Universität Berlin

Partei- und fraktionspolitische Verhandlungslogiken und Entscheidungsmuster im Europäischen Parlament

Prof. Dr. Jürgen MITTAG, Jean-Monnet-Professur, Sporthochschule Köln

Informationskampagnen zu den Europawahlen Dr. Markus WARASIN, Referatsleiter, Generaldirektion Kommunikation, Europäisches Parlament, Brüssel

Strategien der Wahlmobilisierung

Wilhelm LEHMANN, Europäisches Parlament, Brüssel

Interaktions- und Kommunikationsfunktionen des Parlaments

Kontrollfunktionen des Europäischen Parlaments Johann SCHOO, Direktor a.D., Juristischer Dienst, Europäisches Parlament, Brüssel

Das Europäische Parlament und die nationalen Parlamente

Prof. Dr. Gabriele ABELS, Institut für Politikwissenschaft, Eberhard-Karls-Universität Tübingen; Ass.-Prof. Dr. Annegret EPPLER, Universität Innsbruck

Das Europäische Parlament und die Medien Dr. Doris DIALER, Europäisches Parlament; Universität Innsbruck

Das Europäische Parlament und Lobbying

Dr. Christine QUITTKAT, Mannheimer Zentrum für Europäische Sozialforschung, Mannheim 
relative Machtzunahme und Abnahme der Aufmerksamkeit für Kompetenz- und Verfahrensfragen in den ,parlamentsschwachen“ Kompetenzfeldern. Politikfeldübergreifend ist gleichwohl eine gestärkte Politikgestaltungskapazität des Europäischen Parlaments klar gegeben. Besonders seit der im Vertrag von Lissabon sanktionierten Ausdehnung des Anwendungsbereichs für das Mitentscheidungsverfahren auf die Bereiche Inneres und Justiz, Handels-, Agrar- und Fischereipolitik ist eine deutliche Zunahme der Legislativkompetenz in den jeweiligen Parlamentsausschüssen zu verzeichnen. Über 80 Prozent der Gesetzgebungsakte wurden in den vergangenen fünf Jahren in der ersten Parlamentslesung verabschiedet, was auf den ersten Blick auf einen Effizienzgewinn des Legislativverfahrens hinweist. Bei näherem Hinsehen stellt sich aber heraus, dass die informellen Verfahren der Einigung im Trilog länger dauern und damit die Dauer des Verfahrens bis zum Abschluss der ersten Lesung immer weiter zunimmt. Da Trilogverfahren intransparenter als ,normale Verfahren sind und damit einen Verlust an Zurechenbarkeit der Verantwortung für legislative Entscheidungen bedeuten, wäre über eine Rückkehr zum traditionellen Mitentscheidungsverfahren mit drei Lesungen nachzudenken. Alternativ wäre zumindest eine engere Begrenzung des Anwendungsbereichs für Triloge zu bedenken.

\section{Systemgestaltungs- und Wahl-/Rekrutierungs- funktionen}

Als „Schatzkiste konstitutioneller Ideen“ prägt das Europäische Parlament die Entwicklung der Europäischen Union spätestens seit Vorlage des Spinelli-Entwurfs. Im offenen, klar als solchem ausgewiesenen Verfassungsgebungsprozess sowie im verdeckten Verfassungsumsetzungsprozess - mittels interinstitutioneller Abkommen in den Zwischenphasen der Regierungskonferenzen zur Vertragsreform - ist das Parlament seit Jahrzehnten aktiv und recht erfolgreich. Schritt für Schritt hat es sich als
Herausforderungen des Parlaments in zentralen Politikfeldern

Einführung

Dr. Theo SOMMER, Ehrensenator der HelmutSchmidt-Universität, Hamburg

Das Europäische Parlament und die Reform der Wirtschafts- und Währungsunion

René REPASI, Universität Heidelberg

Das Europäische Parlament in der Gemeinsamen Außen- und Sicherheitspolitik

Dr. Anja OPITZ, Akademie für Politische Bildung Tutzing

Das Europäische Parlament und der Raum der Freiheit, der Sicherheit und des Rechts

Dr. des. Daniela KIETZ, Stiftung Wissenschaft und Politik, Berlin

Das Europäische Parlament in der Internationalen Handelspolitik

Prof. Dr. Andreas MAURER, Institut für Politikwissenschaft, Universität Innsbruck

\section{Kommentar}

Prof. Dr. Peter SCHIFFAUER, Europäisches Parlament, Brüssel; Dimitris-Tsatsos-Institut für Europäische Verfassungswissenschaften, Fern-Universität Hagen

\section{Tagungsabschluss}

Dr. Andreas KALINA, Akademie für Politische Bildung Tutzing

Prof. Dr. Andreas MAURER, Institut für Politikwissenschaft, Universität Innsbruck

Bühne und Forum der Verfassungsentwicklung der Europäischen Union etabliert. Spätestens seit dem Verfassungskonvent (2002/03), in dem das Parlament unter anderem die Verbindlichkeit der Charta der Grundrechte der Europäischen Union, eine klare Normenhierarchie sowie die Reform des Gesetzgebungsverfahrens der Europäischen Union initiierte, agiert das Parlament nicht länger als abstrakter, hinter den Kulissen wirkender, sondern als konkreter, mit offenem Visier argumentierender Systemgestalter der Europäischen Union. 
Die gesteigerte Sichtbarkeit des Europäischen Parlaments resultiert jedoch nicht nur aus seiner gewonnenen Systemgestaltungskompetenz, sondern ebenso aus der jüngsten Reform seiner Wahlfunktion. Sowohl in inner-als auch in außereuropäischen Wahlen ist das Parlament aktiv. Die Vergabe von innerparlamentarischen Ämtern erfolgt grundsätzlich durch interne Wahlen und Benennungen im Europäischen Parlament. Der Vertrag von Lissabon verstärkte aber vor allem die zweite, außerhalb des eigenen Institutionenradius liegende Seite der Wahlkompetenz des Europäischen Parlaments. Selbst in parlamentsfremden Bereichen ist das Europäische Parlament heute nicht mehr nur für die Ernennung von Amtsinhabern unterschiedlicher EU-Ämter (mit-)verantwortlich - wie etwa direkt bei der Ernennung des Europäischen Datenschutzbeauftragten oder indirekt über das Anhörungsrecht vor dem Rat bei der Wahl der Direktoriumsmitglieder der Europäischen Zentralbank. Nach Art. 17 Abs. 7 Vertrag über die Europäische Union muss bei der Wahl des Kommissionspräsidenten das Ergebnis der Europawahl ,,berücksichtigt $^{\text {“ }}$ werden. Der Europäische Rat soll dem Parlament einen Kandidaten im Lichte der Parlamentswahlen vorschlagen. In der Praxis bedeutet dies, dass der Wahlkampf 2014 erstmals als personalisierter Wahlkampf mit Spitzenkandidaten der Parteien auf europäischer Ebene geführt wird. Mit den Wahlen des Europäischen Parlaments würde somit nicht mehr nur das Parlament, sondern auch indirekt und in Zusammenarbeit mit dem Europäischen Rat der Kommissionspräsident gewählt. Hieraus resultiert nicht nur eine neue Schlagkraft der Wahlen zum Europäischen Parlament, sondern auch eine gesteigerte Sichtbarkeit des Europäischen Parlaments und der Europäischen Union im Allgemeinen. Das Parlament wird zu einem zentralen Ort - neben dem Europäischen Rat - der Rekrutierungsverhandlungen und erfährt eine nach außen sichtbare Stärkung seiner Wahlfunktion.

\section{Profilbildung im und Wahlen zum Europäi- schen Parlament}

Von den UnionsbürgerInnen werden die Europawahlen allerdings weiterhin als ,Nebenwahlen" empfunden. Daraus resultieren systematische Unterschiede, die zu einer relativ geringen Wahlbeteiligung bei Europawahlen führen. Die auf der Tagung diskutierten Studien zeigen, dass für die Bürger bei den Europawahlen weniger auf dem Spiel steht. Die als gering empfundene Relevanz der Wahlen wird dabei zusätzlich von der geringen Profilierung der Parteien im Wahlkampf verstärkt. Im Vergleich zu nationalen Wahlen, werden die Wahlen zum Europäischen Parlament sowohl hinsichtlich der finanziellen Ausstattung der Kampagnen als auch bezüglich der medialen Berichterstattung deutlich niedriger eingestuft. Aufgrund dessen scheint es nicht erstaunlich, dass Europawahlen den Bürgern irrelevanter und als Nebenwahlen erscheinen.

Mit dem Leitspruch ,This time it's different" versucht die Wahlkampagne des Europäischen Parlaments zur Wahl 2014 genau diese Andersartigkeit der Wahlen 2014 zu thematisieren und zu unterstreichen. Dazu sollen die Ernennung der Spitzenkandidaten der Parteien, eine breitere Werbekampagne und eine Serie europaweit übertragener TV-Duelle der Spitzenkandidaten beitragen. Ob die drei Strategien der Wählermobilisierung - Entwicklung europäischer Politikprogramme, Personalisierung und Politisierung der Europawahlen und Europäisierung des Parteiwettbewerbs - tatsächlich in einer gesteigerten Sichtbarkeit und Wahlbeteiligung resultieren wird, wird sich erst in der Nachwahlanalyse sicher beurteilen lassen.

\section{Kontroll-, Interaktions- und Kommunikations- funktionen des Europäischen Parlaments}

Die wichtigsten Akteure der Kontrollfunktion des Europäischen Parlaments sind seine Aus-

1 Zur Analyse der Europawahlen 2014 vgl. Daniel Göler/Mathias Jopp: Die Europawahl 2014 und das Konzept der Spitzenkandidaten - ein Kommentar, in: integration 2/2014, S. 152-160; Rudolf Hrbek: Europawahl 2014: Kontinuität und neue Facetten, in diesem Band, S. 205-228; Andreas Maurer: Die Kreationsfunktion des Europäischen Parlaments im Spannungsfeld zwischen Politisierungsimpulsen und Systemerfordernissen, in: Zeitschrift für Politik, Vol. 61, Nr. 3/2014, S. 301-327. 
schüsse. Von den Parlamentsausschüssen werden sowohl die Plenumsvorbereitungen als auch die Kontrolle der europäischen Institutionen durch Anhörungen und Befragungen der Institutionen $\mathrm{zu}$ relevanten Themen getätigt. Doch auch anhand der Berichtspflicht des Rates, der Kommission und der Europäischen Zentralbank an das Parlament, aufgrund der Möglichkeit von schriftlichen Anfragen sowie durch seine Haushaltskontrolle des von der Kommission durchgeführten Jahreshaushalts, kann das Europäische Parlament seine Kontrollfunktion aktivieren.

Bereits seit den 1980er Jahren sind Initiativen interparlamentarischer Zusammenarbeit des Europäischen Parlaments mit den nationalen Parlamenten zu konstatieren. Ziel der interparlamentarischen Zusammenarbeit ist primär der gegenseitige Informationsaustausch und damit eine effizientere Wahrnehmung der Kontrollfunktionen des Parlaments. Der Vertrag von Lissabon akzentuierte in diesem Zusammenhang Instrumente und Verfahren sowohl für die nationalen Parlamente als auch für das Europäische Parlament. Gleichwohl wird die Beziehung der beiden Parlamentsebenen (immer noch) eher als „Zweckehe“ denn als „Liebesheirat" beschrieben. Lissabon fungierte zwar als Katalysator der Kooperation, doch neben der Intensivierung der Zusammenarbeit ist auch ein Anstieg der interparlamentarischen Konflikte erkennbar, die auf Konkurrenzdenken um limitierte Ressourcen und Kompetenzen beruhen.

In seiner Kommunikationsfunktion bewegt sich das Parlament im Dreieck zwischen Bürgern, Politik und Medien. Eine supranationalisierte ,europäische Öffentlichkeit“, mit europaweiter gemeinsamer medialer Wahrnehmung, hat sich bis jetzt noch nicht entwickelt. Von europäisierten nationalen Öffentlichkeiten durch die mediale Verarbeitung und Verbreitung europäischer Themen in den Mitgliedstaaten der Europäischen Union kann allerdings gesprochen werden. Hier sind vertikale Europäisierungstendenzen erkennbar bis hin zur regionalen Medienebene ist die eu- ropäische Berichterstattung mittlerweile in Einzelfällen durchgedrungen. Die Kommunikation des Parlaments beruht auf einer individualisierten und einer institutionalisierten Form: zum einen durch die Kommunikationskanäle der Abgeordneten, der Fraktionen, Ausschüsse und europäischen Parteien, zum anderen durch die medialen Kanäle der Generaldirektion Kommunikation des Europäischen Parlaments.

Lobbying, das in der breiten Öffentlichkeit vor allem negativ wahrgenommen wird, bildet ein wichtiges Instrument der Informationsbereitstellung durch Vertreter von Partikularinteressen für Politiker. Lobbying übernimmt damit einen wichtigen Teil der Politikgestaltung auf EU-Ebene. Seit 2011 besteht ein gemeinsames Transparenzregister der Kommission und des Europäischen Parlaments, in dem sich Lobbyisten - Organisationen und selbstständige Einzelpersonen - registrieren sollen, aber nicht müssen. Mit dem Register soll eine gesteigerte Transparenz des europäischen Lobbyismus und ein Verhaltenskodex für Lobbyisten erreicht werden.

\section{Herausforderungen des Europäischen Parla- ments in zentralen Politikfeldern}

Nach der Analyse der zentralen Funktionen des Europäischen Parlaments endete die Tagung mit der Diskussion der zukünftigen Herausforderungen. Beginnend mit der Reform der Wirtschafts- und Währungsunion, in der das Parlament formal zwar nur eine marginale Rolle einnimmt, aber dennoch zur Setzung von Reformanreizen im Stande ist (so zum Beispiel im ersten interinstitutionellen Abkommen mit der Europäischen Zentralbank), sind die Sicherung seiner Budgethoheit durch die Haushaltskontrolle und die Absicherung der Gemeinschaftsmethode als Kerninstrument der Europäischen Union wesentliche Schwerpunkte, auf die sich das neu gewählte Parlament einlassen muss.

In der Gemeinsamen Außen- und Sicherheitspolitik hat sich das Europäische Parlament 
Kompetenzen in der Kontrolle, aber auch in der Politikgestaltung angeeignet. So ist die Hohe Vertreterin der Union für Außen- und Sicherheitspolitik in ihrer Eigenschaft als Mitglied der Europäischen Kommission dazu verpflichtet, das Parlament zu konsultieren und es nicht nur im Nachhinein durch Berichterstattung zu informieren. Daraus resultiert ein faktisches Ex-ante-Untersuchungs- und Konsultationsrecht in denjenigen Feldern europäischen Außenhandelns, die nicht exklusiv unter dem Dach der Gemeinsamen Außen- und Sicherheitspolitik beheimatet sind. Hieraus erwächst dem Europäischen Parlament neben der Möglichkeit von Anfragen an den Rat oder an die Hohe Vertreterin insgesamt ein größeres Gewicht in den mit der Gemeinsamen Außen- und Sicherheitspolitik verknüpften Bereichen der europäischen Außenpolitik (zum Beispiel bei internationalen Abkommen oder Sanktionspolitiken), wobei das Parlament seinen spezifischen Beitrag zu mehr Transparenz und Kohärenz leistet.

Auch im Raum der Freiheit, Sicherheit und des Rechts kam mit dem Vertrag von Lissabon Bewegung ins Spiel. Das Parlament ist mittlerweile zentraler Akteur und Mitentscheider in der Justiz- und Innenpolitik. Der Ausschuss für bürgerliche Freiheiten, Justiz und Inneres ist seit dem Inkrafttreten des Vertrags von Lissabon neben dem Ausschuss für internationalen Handel das am stärksten in der Mitentscheidungspolitik tätige Parlamentsgremium. Neben der angestrebten Wahrung seiner Kontrollfunktion dürften sich die Ambitionen der Abgeordneten verstärken, in der Agendasetzung eine aktivere, die Vormacht des Europäischen Rates hinterfragende Rolle einzunehmen. Die Entscheidungsverfahren wurden durch die Vertragsänderungen und die damit einhergehende Gleichberechtigung des Parlaments de- mokratischer, aber gleichzeitig auch komplexer und konfliktanfälliger. Interinstitutionelle Spannungen und Machtkämpfe zur Kompetenzverteilung und gegenläufige Schwerpunktsetzungen der Institutionen sind die Kehrseite der gesteigerten parlamentarischen Demokratie der Entscheidungsfindung.

Schließlich hat die Beteiligung des Europäischen Parlaments in der internationalen Handelspolitik der Europäischen Union in den vergangenen Jahren einen starken Wandel erfahren. Bis zum Inkrafttreten des Vertrags von Lissabon war die Handelspolitik ein parlamentsfremdes Gebiet, in dem der Handelsausschuss relativ folgenlos kommentieren und kritisieren konnte. Seit Dezember 2009 hat sich die Handelspolitik jedoch zu einem entscheidenden Politikbereich des Parlaments entwickelt: In der autonomen Handelspolitik definiert über den im Sekundärrecht erlassenen Acquis der Normierung eigenständigen, europäischen Außenwirtschaftsrechts - ist der Handelsausschuss nun Mitgesetzgeber mit dem Ministerrat. In der angelaufenen Legislaturperiode des Parlaments stellen sich neuartige Fragen der strategischen Ausrichtung. Hinsichtlich der internationalen Handelsabkommen bleiben nicht nur die Mandatsverfahren, sondern auch die Zuständigkeiten im Ministerrat intransparent, sodass dem Europäischen Parlament oftmals verborgen bleibt, wer eigentlich der konkrete Ansprechpartner ist. Hier sollte das Parlament seine Kontrollfunktionen schärfen und festlegen, ob diese bereits bei der Erstellung von Verhandlungsmandaten für Abkommen oder erst im Nachhinein greifen sollen. Umgekehrt steht das Parlament in der Pflicht, den Umgang mit vertraulichen Dokumenten für und aus den Verhandlungen einer Revision zu unterziehen. 